\title{
Wetting of nanophases: Nanobubbles, nanodroplets and micropancakes on hydrophobic surfaces
}

\author{
Hongjie An ${ }^{\mathrm{a}}$, Guangming Liu ${ }^{\mathrm{b}}$, Vincent S.J. Craig a,b,* \\ a Department of Applied Mathematics, Research School of Physical Sciences and Engineering, Australian National University, Canberra, ACT 0200, Australia \\ ${ }^{\mathrm{b}}$ Hefei National Laboratory for Physical Sciences at the Microscale, Department of Chemical Physics, University of Science and Technology of China, Hefei 230026, PR China
}

\section{A R T I C L E I N F O}

Available online $\mathrm{xxxx}$

\section{Keywords:}

Contact angle

Line tension

Nanobubbles

Nanodroplets

Micropancakes

\begin{abstract}
A B S T R A C T
The observation by Atomic Force Microscopy of a range of nanophases on hydrophobic surfaces poses some challenging questions, not only related to the stability of these objects but also regarding their wetting properties. Spherical capped nanobubbles are observed to exhibit contact angles that far exceed the macroscopic contact angle measured for the same materials, whereas nanodroplets exhibit contact angles that are much the same as the macroscopic contact angle. Micropancakes are reported to consist of gas, in which case their wetting properties are mysterious. They should only be stable when the van der Waals forces act to thicken the film whereas for a gas, the van der Waals forces will always act to thin the film. Here we examine the available evidence and contribute some additional experiments in order to review our understanding of the wetting properties of these nanophases. We demonstrate that if in fact micropancakes consist of a contaminant their wetting properties can be explained, though the very high contact angles of nanobubbles remain unexplained.
\end{abstract}

(c) 2014 Elsevier B.V. All rights reserved.

\section{Contents}

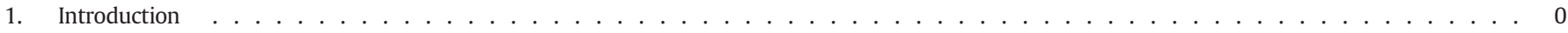

2. Materials and methods $\ldots \ldots \ldots$

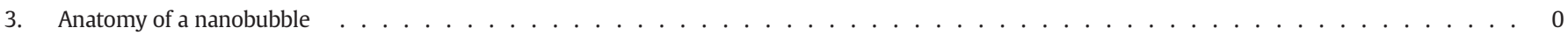

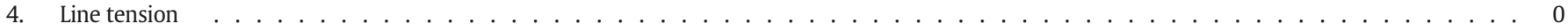

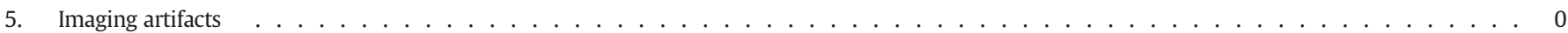

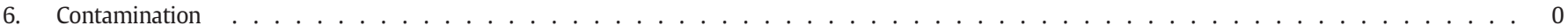

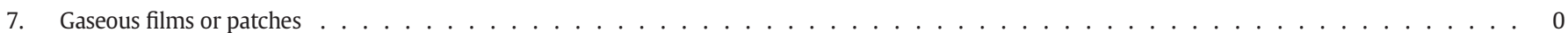

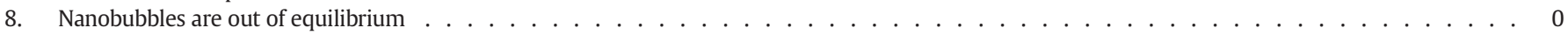

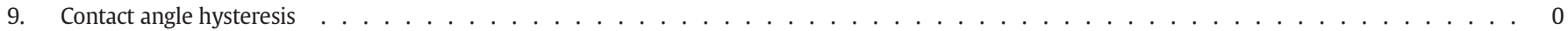

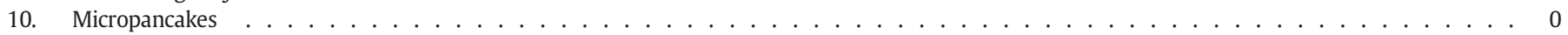

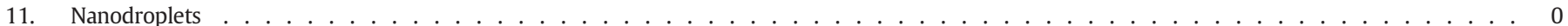

12. Micropancakes and nanodroplets in DMSO . . . . . . . . . . . . . . . . . . . . . . . . . . . . . . . . 0

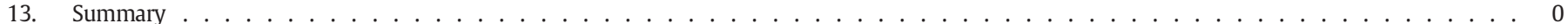

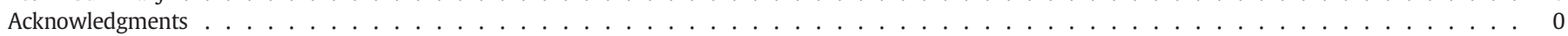

References $\ldots \ldots$

\section{Introduction}

It is now well established that under favorable conditions nanobubbles are present on hydrophobic surfaces in aqueous solutions

\footnotetext{
* Corresponding author at: Department of Applied Mathematics, Research School of Physical Sciences and Engineering, Australian National University, Canberra, ACT 0200 Australia. Tel.: +61 26125 3359; fax: +6126125 0732 .

E-mail address: vince.craig@anu.edu.au (V.S.J. Craig).
}

[1-3], though the acceptance of their counterparts, nanobubbles in bulk [4-7], is yet to gain wide acceptance. Surface nanobubbles are mysterious in two regards [8]. Firstly, theoretical considerations predict that they should dissolve in a few seconds $[9,10]$, which is in contrast to observations that show they are stable from tens of minutes to days [11]. Secondly, the contact angles that nanobubbles make with hydrophobic surfaces are considerably different from macroscopic contact angles measured on the same surfaces [12]. It is important to resolve the contact angle anomaly as wetting at these scales is central to a 
host of phenomena including, flow through porous materials [13], enhanced oil recovery [14], adsorption of emulsion droplets [15], capillary condensation, heat transfer, electrochemistry and heterogenous nucleation. Further, nanobubble researchers would like to determine if the contact angle anomaly is related to the stability of surface nanobubbles [8]. Researchers have examined line tension, contamination, gaseous films and imaging artifacts as possible sources of the anomaly. Here we look at the evidence supporting and opposing these explanations. We also examine the unusual wetting properties of micropancakes [16-19] and perform a series of experiments to reproduce these structures.

\section{Materials and methods}

All water used in these experiments was purified using a coarse filter, a carbon filter and a reverse osmosis filter before being passed through a Millipore gradient system. AR grade ethanol was further purified by distillation before use. Anhydrous DMSO ( $\geq 99.9 \%)$ was supplied by Sigma-Aldrich. For some experiments DMSO was degassed in a round bottom flask equipped with a vacuum valve. The DMSO was frozen and degassed by pumping as the DMSO melted. This freeze-thaw cycle was repeated three times. Glass and plastic syringes were used as specified. Glass syringes were cleaned with ethanol and thoroughly rinsed with water before being dried using a stream of nitrogen gas. The high purity nitrogen gas is obtained as the 'boil-off' from liquid nitrogen storage. Sterile plastic syringes (Terumo) of volume $10 \mathrm{~mL}$ were opened immediately before use and used without cleaning. Plastic syringes were used only once and discarded. The tubing used was Teflon and a glass AFM fluid cell was sealed by a silicone ' $O$ ' ring. The ' $O$ ' ring had been soaked extensively in ethanol before use. In order to form nanobubbles or micropancakes the procedure known as solvent exchange is routinely employed. The AFM fluid cell was first filled with water and the surface imaged as a control. Then, the water was displaced by passing ethanol $(\sim 10 \mathrm{~mL})$ through the cell over a period of approximately $1 \mathrm{~min}$. Again the surface was imaged as a control. The ethanol was then displaced with $10 \mathrm{~mL}$ of water over approximately $1 \mathrm{~min}$ and the features produced were then measured.

A multimode Nanoscope IIIa scanning probe microscope (Bruker Corporation, Santa Barbara, CA) equipped with a vertical engage J scanner was used in tapping mode. A fluid cell with a non-spherical cross section O-ring was used to image nanobubbles. Oxide-sharpened silicon nitride cantilevers (OMCL-TR400PSA, Olympus) with a nominal spring constant of $0.08 \mathrm{~N} / \mathrm{m}$ and nominal resonance of $34 \mathrm{kHz}$ in air were used for imaging in liquids. The cantilevers were cleaned with water vapor plasma using a custom-built plasma reactor ( $30 \mathrm{~W}, 30 \mathrm{~s})$. Cantilevers (Tap300Al-G, Budget Sensors) with a nominal spring constant of $40 \mathrm{~N} / \mathrm{m}$ were used for imaging surfaces in air.

The macroscopic contact angle of polydimethylsiloxane (PDMS) oil (Silicone oil, viscosity $100 \mathrm{mPa} . \mathrm{s}\left(20^{\circ} \mathrm{C}\right)$, Sigma-Aldrich) on a HOPG surface was measured by an optical contact-angle goniometer with automatic dispenser (CAM 200, KSV Instruments Ltd.). Sample liquid was expelled from a pre-cleaned glass and Teflon syringe onto newly cleaved HOPG. The profile of the droplet was recorded using a video camera.

\section{Anatomy of a nanobubble}

Nanobubbles are gaseous domains that are typically tens to hundreds of nanometers in radius but only $10-100 \mathrm{~nm}$ high. Atomic Force Microscope (AFM) images reveal that they form spherical caps. This is consistent with considerations of the pressure inside the bubble, where the Laplace pressure describes the local curvature of the interface. A schematic of a nanobubble is provided in Fig. 1, showing the salient features. Very close to the substrate, the shape of the interface should deviate from that of a spherical cap due to the action of surface forces $[20,21]$, but this has not been observed due to limitations of the

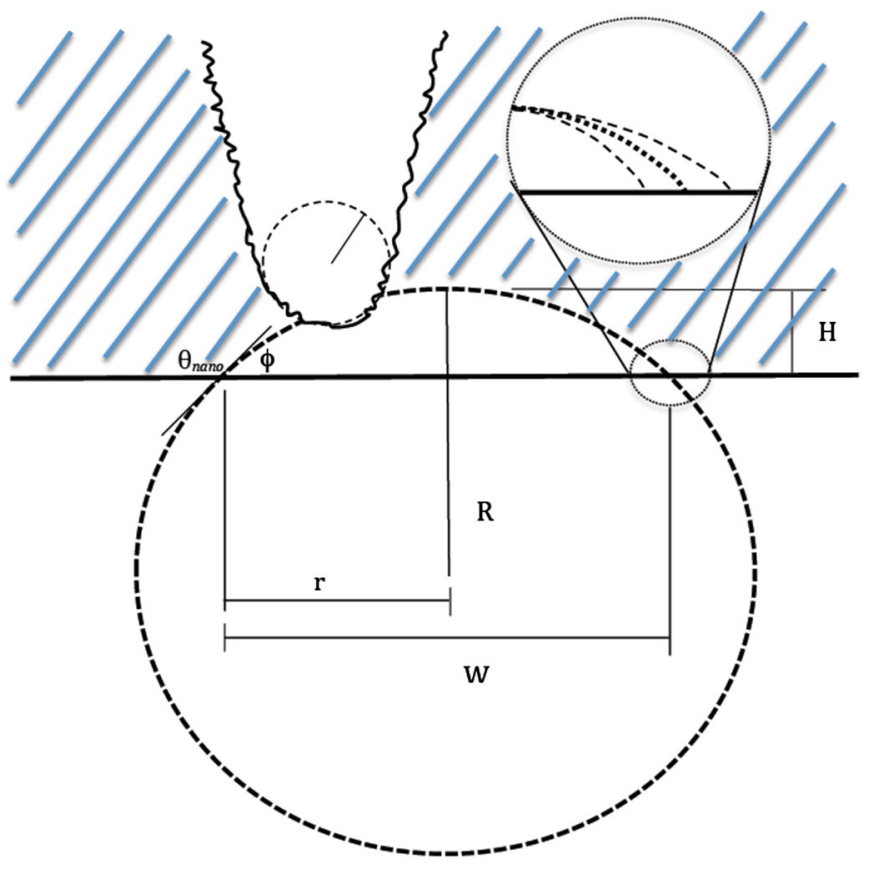

Fig. 1. Schematic of a nanobubble and the tip of an AFM cantilever. The nanobubble is a spherical cap of radius $R$, with width $W$, and height $H$, at the apex. It makes an internal angle of $\phi=\sin ^{-1}(W / 2 R)$ with the hydrophobic substrate. The contact angle $\theta_{\text {nano }}=180^{\circ}$ - $\phi$ typically exceeds the macroscopic contact angle, $\theta_{\infty}$, on the same surface by $20^{\circ}$ to $60^{\circ}$ and is by convention measured through the more dense aqueous phase. The radius of the contact line is $r$ and equals $W / 2$. The shape of the AFM tip can be approximated as spherical. The force applied to the nanobubble by the tip can either deform the nanobubble or penetrate the nanobubble, leading to imaging artifacts. Near the threephase line the surface forces will cause the interface to deviate from that of a spherical cap as depicted in the inset. This deviation cannot currently be discerned by AFM.

imaging technique. See the magnified area in Fig. 1. The finite size of the AFM cantilever tip, the deformability of the interface, the difficulty in imaging surfaces at an angle to the cantilever, the small dimensions involved and the roughness of the surface combine to confound attempts to precisely follow the shape of the nanobubble adjacent to the three phase line (TPL) with nanometer precision. As there are so many challenges that need to be addressed to resolve this, it is unlikely that this will be achieved in the near future by AFM. This is unfortunate, as knowledge of the precise shape of the nanobubble near the TPL would enable the magnitude of the line tension to be calculated by means complementary to the analysis of contact angle as a function of contact radius, $r[20,21]$ and reveal details of the surface forces.

Ignoring the region near the TPL the remainder of the bubble can be fit to a spherical cap, or a section though the apex of the bubble can be fit with a circle, in order to obtain both the radius of curvature and the contact angle. Both methods have been employed. When fitting a section it is generally taken from the fast scan direction of the image as this data is least likely to be influenced by drift, or artifacts associated with the rendering of the image [12,22]. In comparison, fitting a spherical cap has the advantage of making use of more data points [23-26], but is more susceptible to the influence of drift and errors associated with data manipulation used to render the image. Both methods require that the height of the substrate is determined and this is not always straightforward due to convolution of the tip. For a system at equilibrium a minimum in the surface energy is described by Young's equation.

$\gamma_{s v}-\gamma_{s l}+\gamma_{l v} \operatorname{Cos} \theta=0$

where $\gamma_{s v}$ is the solid-vapor surface tension, $\gamma_{s l}$ is the solid-liquid surface tension, $\gamma_{l v}$ is the liquid-vapor surface tension and $\theta$ is the contact angle that the droplet makes with the solid. We note here that in the literature the contact angle of surface nanobubbles is reported using 
both the internal angle of the spherical cap, measured through the gas phase, $\phi$, and the complementary angle, measured through the aqueous phase, $\theta_{\text {nano }}$ (see Fig. 1 ). These angles are of course directly related as $\theta_{\text {nano }}+\phi=180^{\circ}$, so one description is readily converted to the other. Here we prefer to use the angle measured through the aqueous phase, $\theta_{\text {nano }}$, as this is consistent with the tradition of reporting the contact angle through the denser phase in oil/water/solid systems. Additionally this is consistent with how the contact angle of a macroscopic water droplet in air is reported. Consistently investigators have found that the contact angle of a nanobubble on a hydrophobic surface, $\theta_{\text {nano, }}$, differs considerably from the macroscopic contact angle obtained using the same materials, $\theta_{\infty}$. When the contact angle is measured through the aqueous phase, $\theta_{\text {nano }}>\theta_{\infty}$ and the difference is usually in the range of $20^{\circ}$ to $60^{\circ}$.

\section{Line tension}

As $\theta_{\text {nano }}>\theta_{\infty}$, a nanobubble is flattened and the TPL is longer than it would be if it were a bubble of the same volume exhibiting a macroscopic contact angle. This immediately requires that a negative line tension be considered as playing a role in the contact angle anomaly [12]. Line tension can be either positive or negative and will vary depending on the nature of the interfaces [20]. Theoretical considerations indicate that the magnitude of the line tension should be of the order of $10^{-11} \mathrm{~N}[20,27-30]$, though experiments find values of the line tension that differ by 5 orders of magnitude [28,29]. So line tension is a controversial topic. As the TPL is longer than expected for a nanobubble, the implication is that the line tension should be negative if it is to resolve the contact angle anomaly. That is the TPL lowers the energy of the system. Note, a positive line tension would decrease $\theta_{\text {nano }}$ and provide a lower limit to the size of a nanobubble that can adhere to a surface [31], as upon reducing the contact perimeter the contact angle would eventually become zero, which implies complete wetting by the aqueous phase. Therefore, either a positive or negative line tension may be of significance for understanding nanobubbles at surfaces.

The influence of the line tension on the contact angle requires modification of Young's equation to include the energy associated with the TPL [30].

$\gamma_{s v}-\gamma_{s l}+\gamma_{l v} \operatorname{Cos} \theta_{\text {nano }}+\frac{\tau}{r}=0$

Which when combined with Eq. (1) gives;

$\operatorname{Cos} \theta_{\text {nano }}+\frac{\tau}{\gamma_{l v} r}=\operatorname{Cos} \theta_{\infty}$

Eq. (3) provides a convenient means to experimentally determine the line tension from a linear fit of $\operatorname{Cos} \theta_{\text {nano }}$ versus $r^{-1}$ and an extrapolation of this data to large $r$ should be in agreement with the macroscopic contact angle, if the trend observed and the contact angle anomaly are due to line tension. However, the experiments do not provide a consistent measure of the line tension. In some cases the data provides a linear correlation $[12,32]$ whereas in other work no such determination is possible [22-24]. Even in cases where a line tension is resolved it is found that the line tension is not sufficient to explain the contact angle anomaly $[12,32]$. Moreover, the line tension should change with temperature typically reducing as the temperature increases [21], but no strong temperature dependence is seen for the contact angle of nanobubbles. Despite the ongoing disagreement as to the magnitude of the line tension, we can conclude from the available data on nanobubbles that the line tension is not the cause of the elevated contact angle observed for nanobubbles.

\section{Imaging artifacts}

The Atomic Force Microsope necessarily applies a small but finite force to the surface when imaging. The imaging force will deform the nanobubble and this will contribute to an error in the image. This error will typically reduce the apparent height of the nanobubble and may therefore influence the measured contact angle. Additionally, the interaction with the nanobubble will depend on the surface properties of the nanobubble. When the surface of the bubble is clean, the AFM tip may penetrate the nanobubble interface, whereas when the cantilever and tip are coated with a surfactant, the interface will deform rather than be penetrated [22]. By adjusting the imaging conditions to minimize the forces applied to the bubble the degree of deformation can be reduced. This has been studied in some detail and when small imaging forces are used the influence of deformation or penetration on the contact angle is an overestimation of less than $10^{\circ}[22,25,26]$.

AFM cantilevers are equipped with sharp tips, which are used to render the image. Ideally the tip would be infinitely sharp and have no influence on the shape of the objects being imaged. In reality the tip has a finite size, which is usually described as an effective radius of curvature (see Fig. 1). The image obtained is in fact a convolution of the feature with the tip shape. The influence of the blurring due to finite tip size will influence the image most where the topography changes rapidly, this is around the TPL for a nanobubble, whereas it will have comparatively little effect at the apex of the nanobubble. Provided the shape of the tip or the effective radius of curvature of the tip is known, it is relatively straightforward to correct for this error, particularly for nanobubbles where the shape of the object is known to be a spherical cap [24]. While care needs to be taken when capturing images of nanobubbles and interpreting the images, it is clear that imaging artifacts are not the cause of the observed contact angle anomaly.

\section{Contamination}

It is well known that contact angle measurements are acutely sensitive to surface contamination. Therefore it has been suggested that the anomalous contact angles observed for nanobubbles may be due to the presence of contamination, such as insoluble organic materials [11] or siloxane [23] that originates from the packaging used for transporting cantilevers. It is of course very difficult to prove that measurements made in aqueous systems are free from contaminant. A more fruitful approach is usually to add a candidate contaminant and observe any change that takes place. Nanobubbles have been imaged in surfactant solutions $[22,33]$. Surfactants are highly surface active and will therefore displace contaminants from the interface. If contaminants influence the contact angle significantly, large changes in the contact angle should be observed when surfactant is added. Three classes of surfactant, cationic, anionic and non-anionic, were studied by Zhang et al. [22]. In all cases only minor changes in contact angle were observed that were similar to the changes observed for the macroscopic system. That is, large differences in $\theta_{\text {nano }}$ and $\theta_{\infty}$ remained. Also, the addition of electrolyte and variations in $\mathrm{pH}$ have minimal influence on the observed contact angles [22]. In order to directly address the possibility that PDMS contamination is responsible for the contact angle anomaly, Song et al. [24] exposed nanobubbles to PDMS and observed no significant changes in contact angle. The observation of the enhanced contact angle has been reported by many different laboratories and it is unlikely that the same contamination problems are present from laboratory to laboratory especially as many of the groups have a wealth of experience in working with clean interfaces in aqueous systems and a variety of techniques are used to clean the cantilevers, substrates, solutions and other equipment employed. Moreover, when we consider macroscopic measurements of water droplets on hydrophobic surfaces it is very rare that contact angles of $120^{\circ}$ or higher are obtained on smooth surfaces and such high contact angles usually involve perfluorinated surfaces. There are no known smooth surfaces that produce macroscopic water 
contact angles in air of $150^{\circ}$ or greater at room temperature, as is observed for nanobubbles. In the absence of a candidate contaminant that could lead to such high contact angles, it is safe to rule out contamination as the cause of the contact angle anomaly. As even if contamination is present in sufficient quantities, it is unlikely to produce the very high contact angles observed.

\section{Gaseous films or patches}

It has been proposed that the anomalously high contact angles of nanobubbles is related to the presence of a gaseous layer that has accumulated at the hydrophobic interface [34,35], or in isolated regions adjacent to the nanobubbles [36]. The presence of a gaseous film on a hydrophobic surface in the absence of surface nanobubbles is not supported by ellipsometric measurements [37] nor studies using $\mathrm{CO}_{2}$ exchange [38] which show no evidence of gaseous $\mathrm{CO}_{2}$ films on the surface in the absence of nanobubbles. However a neutron reflectivity study finds evidence of a depletion layer on polystyrene surfaces that is several nanometers thick [39] and one group has reported AFM images which are claimed to be molecular layers of nitrogen [40]. One could argue that the gas films interact with the nanobubbles and are only present when nanobubbles are present on the surface, but it remains to be seen how the presence of such gas films leads to an elevation in the contact angle and why they do not play a role in macroscopic measurements of contact angle. Pre-wetting films are known to influence the contact angle for liquids in air, however the dispersion forces act to thicken these films for a liquid in air, making them thermodynamically favorable. In contrast, for a gaseous pre-wetting film in water the dispersion forces act to thin the film, making them energetically unfavorable. If patchy gas films are present several questions arise. If patchy gaseous films come into contact with the TPL of the nanobubble why are they not consumed by it or alternatively why do they not consume the nanobubble? Alternatively, if the gaseous patches are not in contact with the nanobubble how do they influence the contact angle? Work on rough surfaces has established that the local environment of the TPL is what determines the contact angle [41], so the effect must be rendered directly in this zone not in a region remote from the TPL.

\section{Nanobubbles are out of equilibrium}

The Young equation is strictly only correct for systems in equilibrium. It is known that for volatile liquids the measured contact angle can differ considerably between that examined in a saturated vapor and that in an unsaturated vapor, as the degree of saturation will strongly influence the thickness of a prewetting film, which is controlled by the dispersion forces [42]. For a macroscopic liquid droplet on a hydrophobic substrate, equilibrium requires a constant temperature and gas saturation. In contrast, a nanobubble on a surface is at best in an unstable equilibrium and generally not in equilibrium; rather it will either dissolve or grow depending on the degree of saturation of the solution near the nanobubble. One might therefore imagine that this could lead to an anomalous contact angle. Nanobubbles are observed for long periods of time (hours or even days) and therefore they appear to obtain a quasi-equilibrium, under such conditions, non-equilibrium effects on the contact angle will be insignificant [43].

\section{Contact angle hysteresis}

Contact angle measurements usually reveal some degree of hysteresis as the measured contact angle for an advancing and a retracting threephase line differ. For a nanobubble that is pinned, the contact angle will increase if the bubble is shrinking. Therefore as a pinned nanobubble dissolves it should exhibit an inflated contact angle and this may provide an explanation for the anomalously high contact angles observed. However, nanobubbles are also observed to grow [38] and therefore the same considerations lead to the expectation that these bubbles will exhibit a reduced contact angle. We find that this is not the case, rather shrinking and growing bubbles exhibit similar contact angles, that are both much higher than their macroscopic equivalents [38]. Furthermore, in some cases it is clear that the nanobubbles are not pinned as the TPL moves during growth or shrinking, or under mechanical action of the cantilever, yet these nanobubbles also exhibit anomalously high contact angles [38].

\section{Micropancakes}

A consideration of the wetting of nanophases is not complete without consideration of "micropancakes". Micropancakes have been reported on some surfaces following ethanol exchange and are distinguished by their unusual morphology in that they may extend laterally by several microns but are only a few nm thick $[16,18,44-46]$. They are called 'pancakes' as the thickness is uniform up to the edge of the structure and therefore the morphology is very different from nanobubbles, which are spherical caps. Micropancakes are reported as being gaseous objects $[16,18,45,46]$. De Gennes has described the formation of wetting pancakes of fluids, which have a similar morphology and are notable in that they are thicker than the molecular scale [42]. These objects are stabilized by an interaction energy that is positive, driven by Van der Waals forces that act to grow the wetting film (negative Hamaker constant for the substrate-liquid-gas system). The magnitude of the Van der Waals forces determines the thickness of the pancake. Micropancakes of gas cannot be stabilized in the same manner as the low density of the gaseous phase ensures that the van der Waals forces act to shrink the pancake (positive Hamaker Constant for the substrate-gas-liquid system) and the interaction energy is negative. That is, the theory of de Gennes [42] that explains the formation of a pancake for a liquid phase on a surface in air, also predicts that a micropancake consisting of a gas phase in liquid should not exist.

Unlike nanobubbles, it has not been clearly established that micropancakes are actually gaseous, though it has been reported that they are unstable in unsaturated solutions [46]. However, the observation that nanobubbles have been observed sitting on top of micropancakes [46] suggests that they are not gaseous. We therefore consider that they may in fact consist of a liquid. In our lab we had never observed micropancakes, even though we used the same solvent exchange technique employed by others and routinely employed HOPG surfaces. This led to the hypothesis that micropancakes consisted of a liquid contaminant introduced during solvent exchange. The ethanol used in solvent exchange is not a candidate for forming micropancakes, as ethanol is fully miscible with water. It is unlikely that such a contaminant is introduced in the water or the ethanol and therefore we considered the tubing and the syringes.

Most laboratories use Teflon tubing as we do, which we believe does not introduce contaminants based on extensive use in ellipsometric [47-51] and surface force studies [52,53]. Using Teflon tubing we are able to flow solutions into a reflectometer cell for hours without any deviation from the baseline [49,54]. Some laboratories may employ silicone tubing, which could possibly introduce PDMS as a contaminant, but we feel this is unlikely to be present in significant quantities, especially as the liquid is in contact with the tubing for a short period of time and the tubing would normally have previously been cleaned by exposure to water and ethanol which should leach any labile contaminant. We do note that this may be an issue when surfactant solutions are employed which can result in leaching of contaminants from tubing. An issue that is resolved by replacing the tubing with teflon [54]. We only use glass syringes in our laboratory and these have been previously cleaned numerous (>20) times using caustic solutions and highly purified ethanol. Therefore, any contaminants present during manufacture have been removed. However, we understand that in some laboratories sterilized disposable plastic syringes are used for handling liquids used in AFM experiments. During the manufacturing process, syringes are 


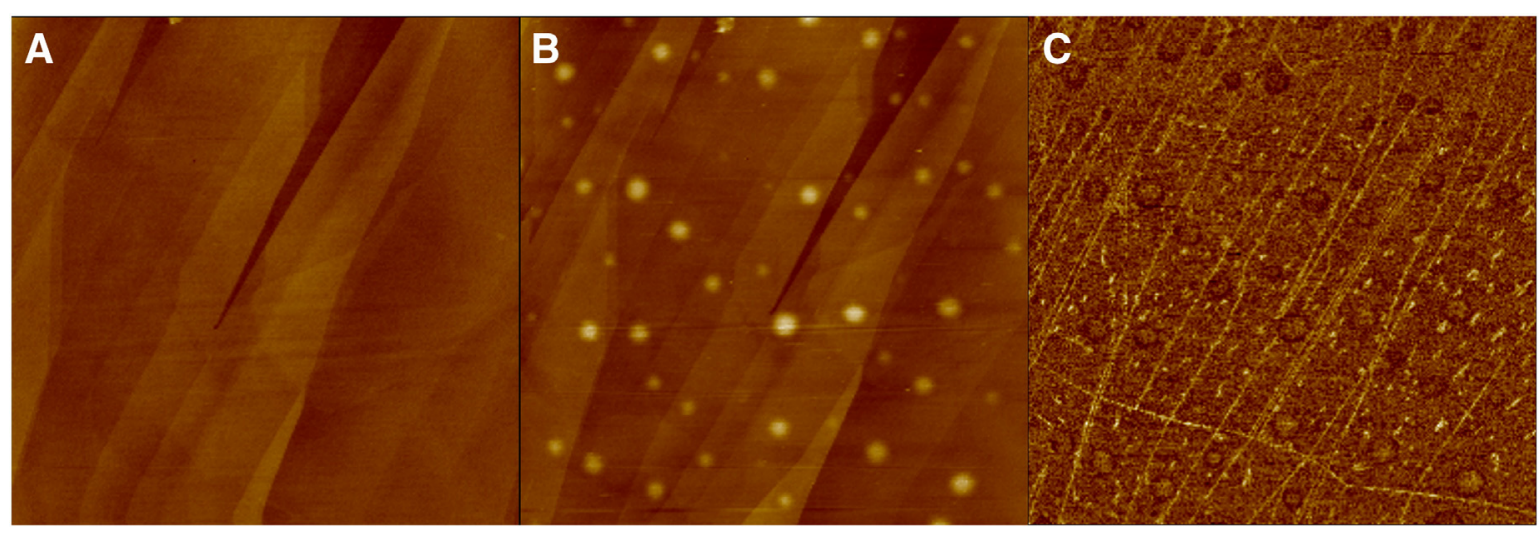

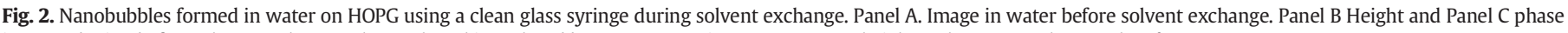
images obtained after solvent exchange where ethanol is replaced by water. Scan size: $5 \mu \mathrm{m} \times 5 \mu \mathrm{m}$, height scale: $20 \mathrm{~nm}$. Phase scale: $2^{\circ}$.

coated with an emulsion of PDMS which acts to lubricate the barrel of the syringe [55]. This may be achieved by direct application of the pure polymer or as a water-soluble emulsion (at $35 \mathrm{wt} . \%$ known as Dimethicone NF emulsion or at 50 wt.\% medical grade dispersion). PDMS has a very low solubility in water and ethanol, but in the emulsified form, which is applied to the syringe, it may be solubilized in aqueous and ethanolic solutions readily.

To test whether transfer of the PDMS from a disposable plastic syringe could result in micropancake like features we conducted the following experiments. Firstly we used the normal solvent exchange procedure with a glass syringe and a HOPG surface. Nanobubbles were observed following solvent exchange as shown in Fig. 2. We then removed the nanobubbles by replacing the water with ethanol. The same solvent exchange procedure was then followed but using a freshly opened disposable plastic syringe instead of a glass syringe. We found that the modified procedure produced micropancakes in the AFM image with a height of $4.5 \mathrm{~nm}$ and large lateral dimensions that are pinned by the steps in the HOPG as previously observed, see Fig. 3. We believe that the observed features are contaminant from the plastic syringe and suggest that the most likely candidate is PDMS as it is added during manufacture. Examination of the contact angle of PDMS oils on HOPG in air resulted in contact angles that were initially $4-6^{\circ}$ and reduced steadily to $\sim 0^{\circ}$ over $30 \mathrm{~s}$. A completely wetting phase is consistent with the formation of micropancakes.

In a separate experiment using a plastic syringe in which the ethanol was left in the fluid cell for $1 \mathrm{~h}$ before being displaced by water we observed a far greater number of objects that looked only slightly different from nanobubbles in the height image as shown in Fig. 4A. However, the phase image suggested that they were not nanobubbles, which should appear as bright objects in the phase image. These objects were dull and appeared similar to the surrounding material, see Fig. 4B. To test whether these were nanobubbles or other material we removed the water and dried the samples and imaged them again. An example of the images obtained is shown in Fig. 5. The objects remained and formed a film on the surface proving that they were not nanobubbles which of course cannot exist when the liquid is removed and should leave no residue on the surface. We note a very recent publication has found that PDMS contamination can also arise from disposable needles and that the use of disposable needles and plastic disposable syringes in nanobubble studies appears to have been the norm in some research groups [56]. Therefore a number of publications both on micropancakes and nanobubbles may have been impacted. We note that not all nanobubble studies are impacted and the existence of gaseous nanobubbles should not be questioned.

\section{Nanodroplets}

Using a modification of the solvent exchange technique that is employed to produce nanobubbles, similar sized features consisting of oil can be produced. These nanodroplets $[57,58]$ can be imaged by AFM in the same manner as nanobubbles. It is noteworthy that the nanodroplets exhibit contact angles that are similar to bulk contact angles for the same materials - that is they do not exhibit a contact angle anomaly [57]. We note that these systems like nanobubbles are

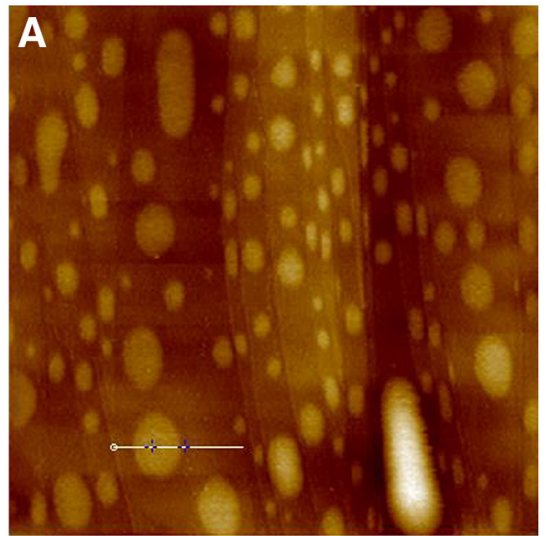

B

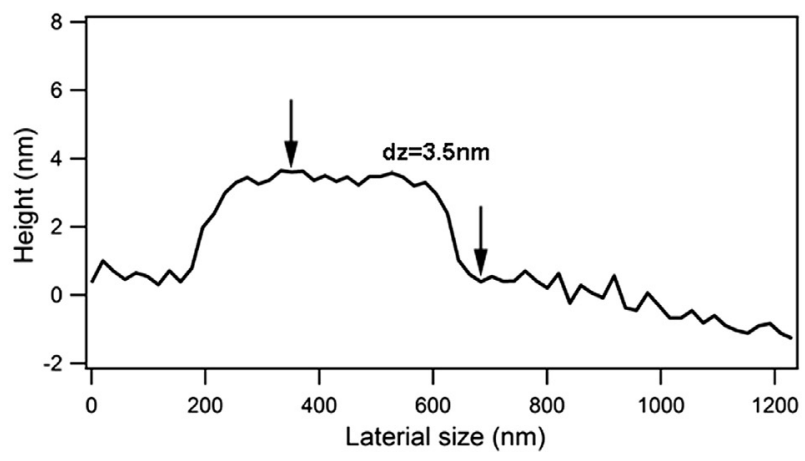

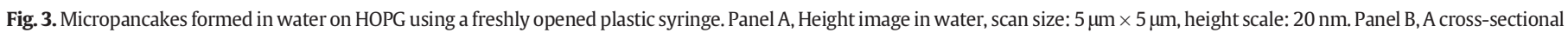
profile of the micropancake at the position indicated in panel A. 

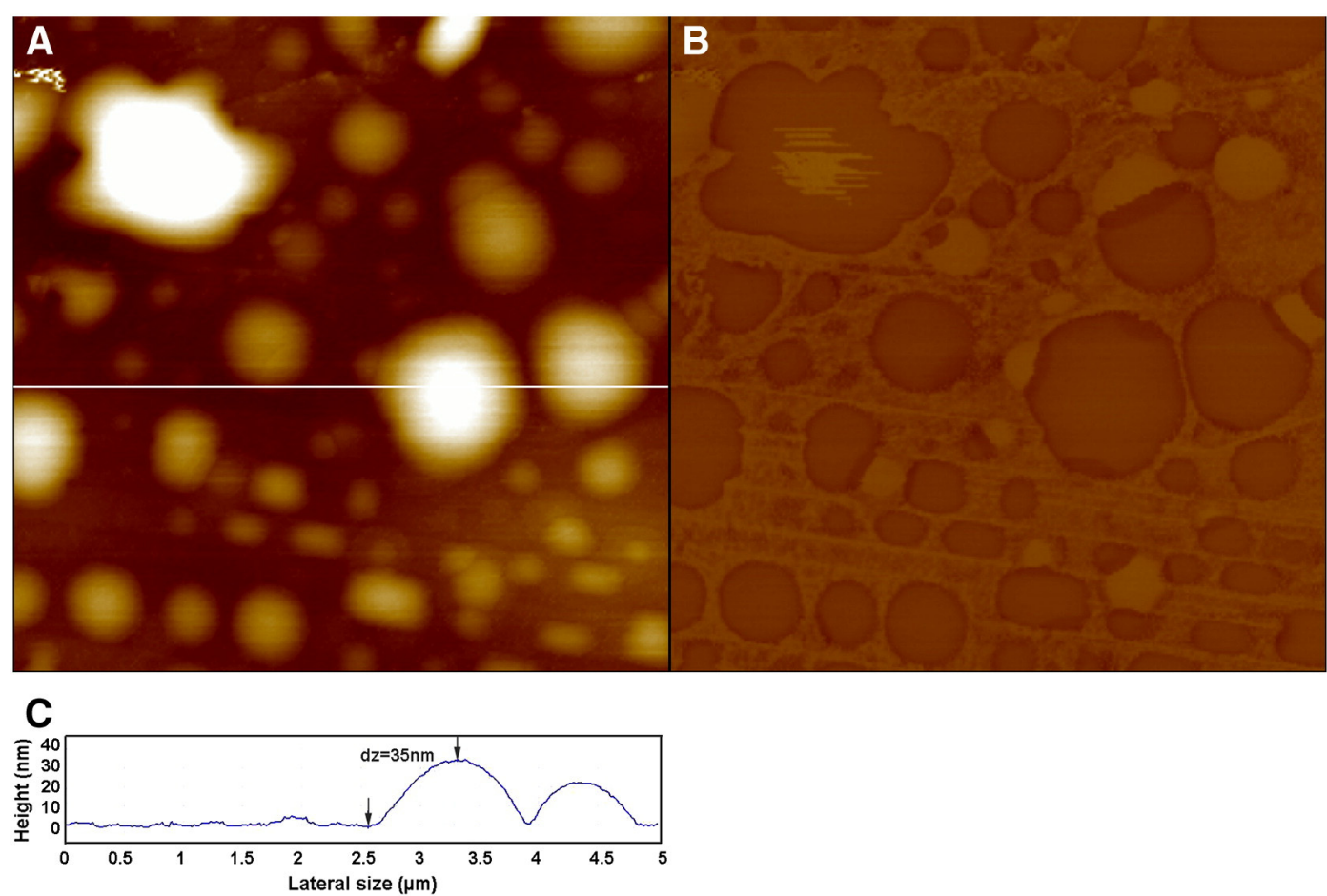

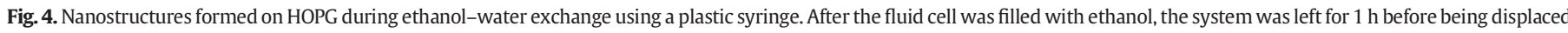

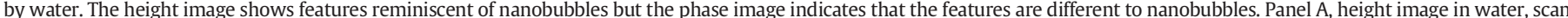
size: $5 \mu \mathrm{m} \times 5 \mu \mathrm{m}$, height scale: $50 \mathrm{~nm}$. Panel B, phase image in water, scan size: $5 \mu \mathrm{m} \times 5 \mu \mathrm{m}$, phase scale: $20^{\circ}$. Panel C, a cross-sectional profile at the position indicated in panel A.

likely to have the same level of contamination, exhibit similar imaging artifacts, exhibit contact angle hysteresis, are subject to line tension and are also out of equilibrium. This provides further evidence that these issues are unlikely to be the cause of the contact angle anomaly observed for nanobubbles. It does strongly suggest that the presence of a gaseous phase is a prerequisite for the contact angle anomaly.

\section{Micropancakes and nanodroplets in DMSO}

We also employed dimethylsulfoxide (DMSO) as an alternative solvent to water in solvent exchange experiments. Using a plastic syringe we obtained images of micropancakes on HOPG in DMSO as shown in
Fig. 6. The micropancakes changed significantly when left overnight as they underwent a ripening process that resulted in less but larger micropancakes, though the surface coverage was almost unchanged at $21 \%$ and $20.5 \%$ respectively. Nanobubbles were observed to form after 1 day, see Fig. 7. The nanobubbles appear to sit on top of the micropancakes as reported previously [46]. Nanobubbles are routinely removed by introducing degassed solvent, therefore we investigated the influence of degassed DMSO on the nanobubbles and micropancakes. This is shown in Fig. 6c. The nanobubbles dissolved, but the micropancakes were stable when degassed solvent was introduced into the cell. This is evidence that the micropancakes we observe are not gas filled, rather we believe that they are PDMS from the plastic syringe, as
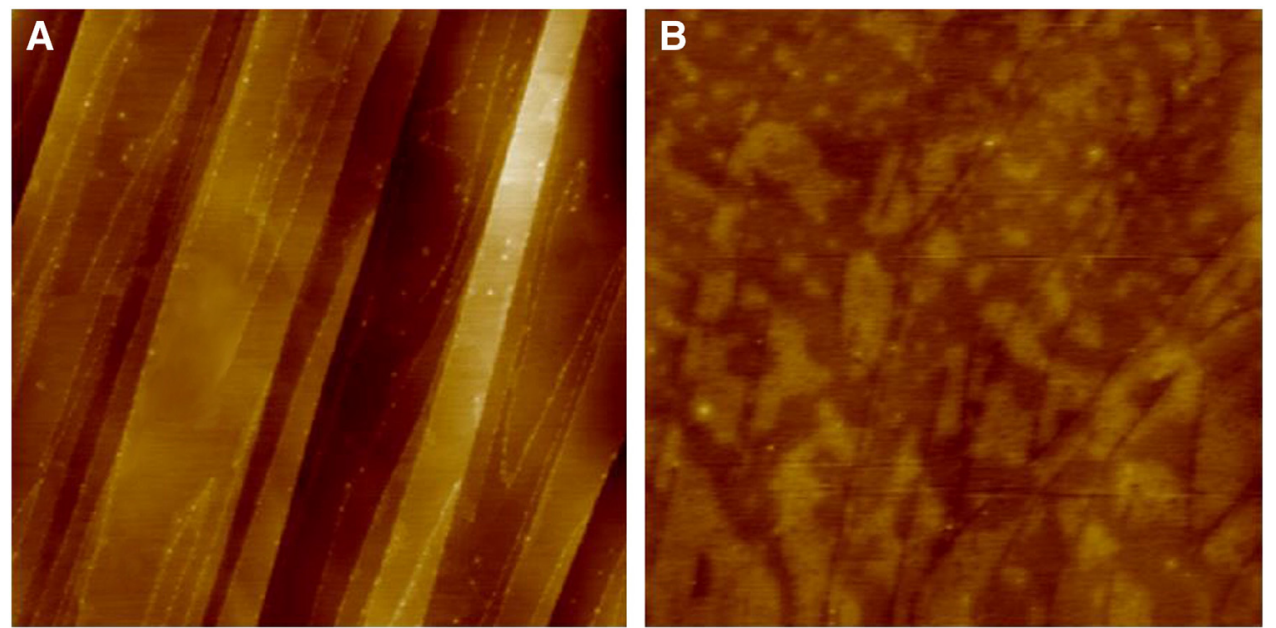

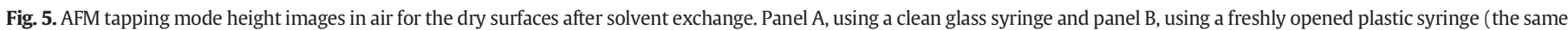
surface as shown in Fig. 4). Scan size: $5 \mu \mathrm{m} \times 5 \mu \mathrm{m}$, height scale: $20 \mathrm{~nm}$. Showing a dried film, about $1.2 \mathrm{~nm}$ in height. 


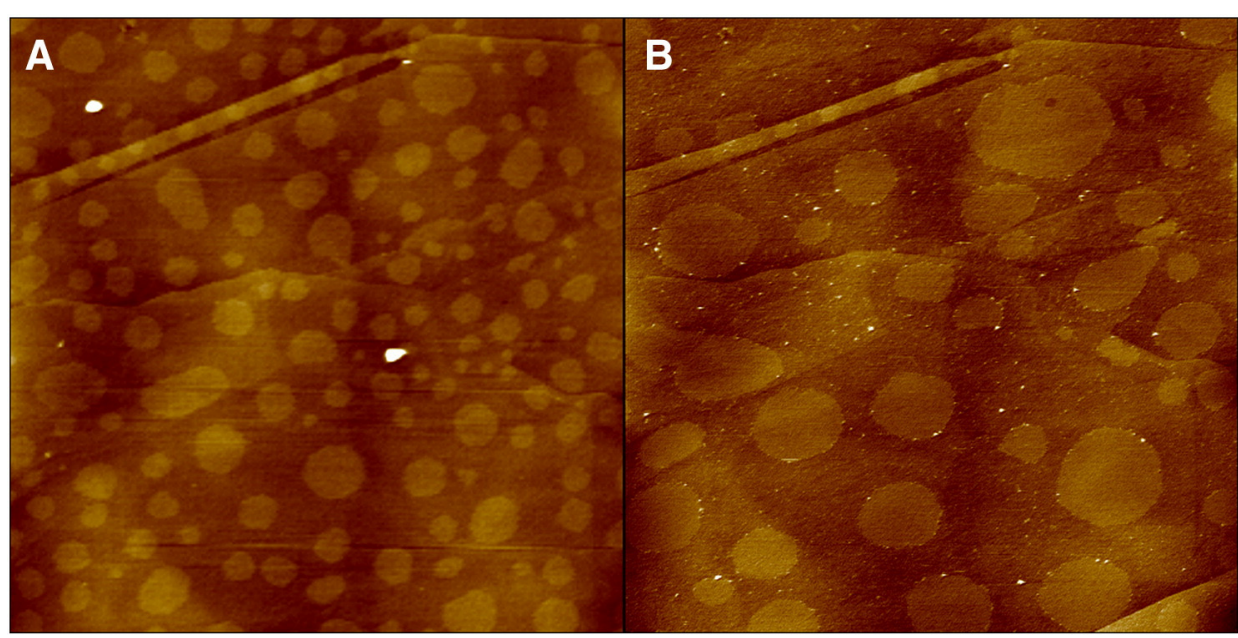

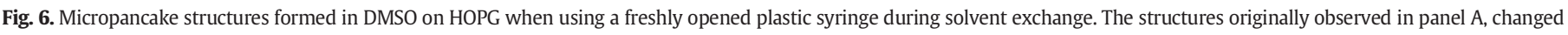
overnight. See panel B. Scan size: $5 \mu \mathrm{m} \times 5 \mu \mathrm{m}$, height scale: $10 \mathrm{~nm}$.

discussed above. In Fig. 8 we present height and phase images in which a nanobubble has moved under action of the AFM cantilever tip. The observation that nanobubbles can be relocated by the AFM tip perturbation and still sit on the surface of micropancakes is consistent with the nanobubble being gaseous and the micropancake being a liquid.

\section{Summary}

We have investigated possible causes of the anomalously high contact angles observed for nanobubbles on hydrophobic substrates. We have considered line tension, imaging artifacts, surface contamination, gaseous films, non-equilibrium measurements, contact angle hysteresis and pinning. We feel that we can rule out all of these mechanisms using a variety of arguments, with the exception of gaseous films. Though we cannot discount the role of gaseous films in elevating the contact angle, there is as yet insufficient evidence to conclude that this is the cause of the contact angle anomaly. Rather the anomaly remains just that and requires further examination and explanation.

On the matter of micropancakes, we have shown that we can obtain micropancakes when a plastic syringe is employed but not when a glass syringe is employed. The evidence strongly suggests that the micropancakes we observe are not gaseous but consist of PDMS lubricant applied to the syringe. We do not claim that in all cases micropancakes are due to PDMS contamination, but advise that care should be taken to avoid the possibility of PDMS contamination and warn against using plastic syringes in any AFM studies. In cases where micropancakes consist of PDMS, their stability is not surprising and their wetting properties are well described by the deliberations of de Gennes on "pancakes".

We advise that plastic syringes should not be used in surface studies and that new glass syringes are extensively cleaned using surfactant, caustic solutions and highly purified ethanol before they are first used. Additionally, we suggest that in future publications on nanobubbles and micropancakes, researchers report the syringes and tubing employed to exchange the fluids. When micropancake features are observed, we suggest drying the sample and re-imaging, to determine if residue left on the surface that would suggest that the experiment was contaminated. As in all surface studies extreme care should be taken to minimize the presence of contamination and controls performed to ensure that the results are not influenced by contamination.

\section{Acknowledgments}

We are pleased to be able to contribute to this special issue dedicated to Professor Reinhard Miller. His expositions on wetting are always insightful, accompanied by humor and delivered with grace. Through him, many people have enjoyed new insights into the deep beauty of droplets and have been inspired to look more closely at wetting phenomena. This research was supported under Australian Research Council's Discovery Projects funding scheme (project number DP110101936).

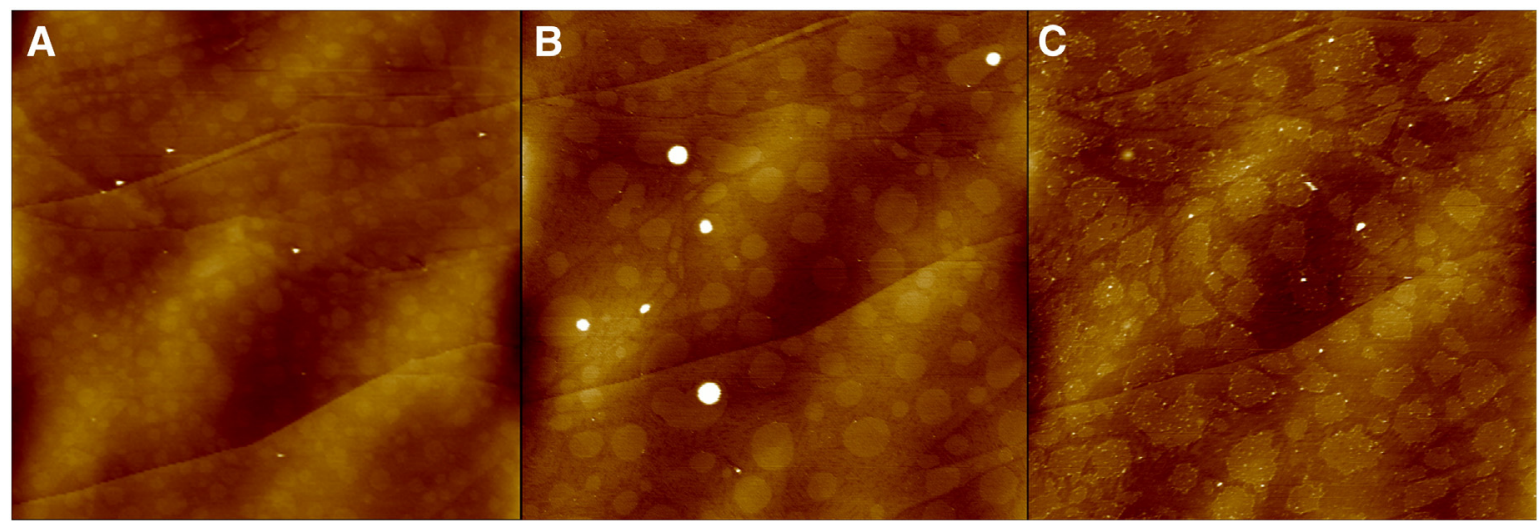

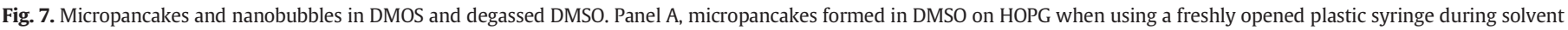

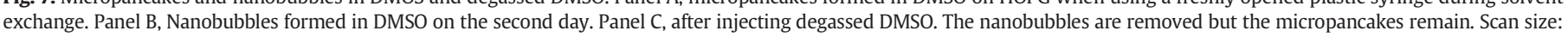
$10 \mu \mathrm{m} \times 10 \mu \mathrm{m}$, height scale: $20 \mathrm{~nm}$. 

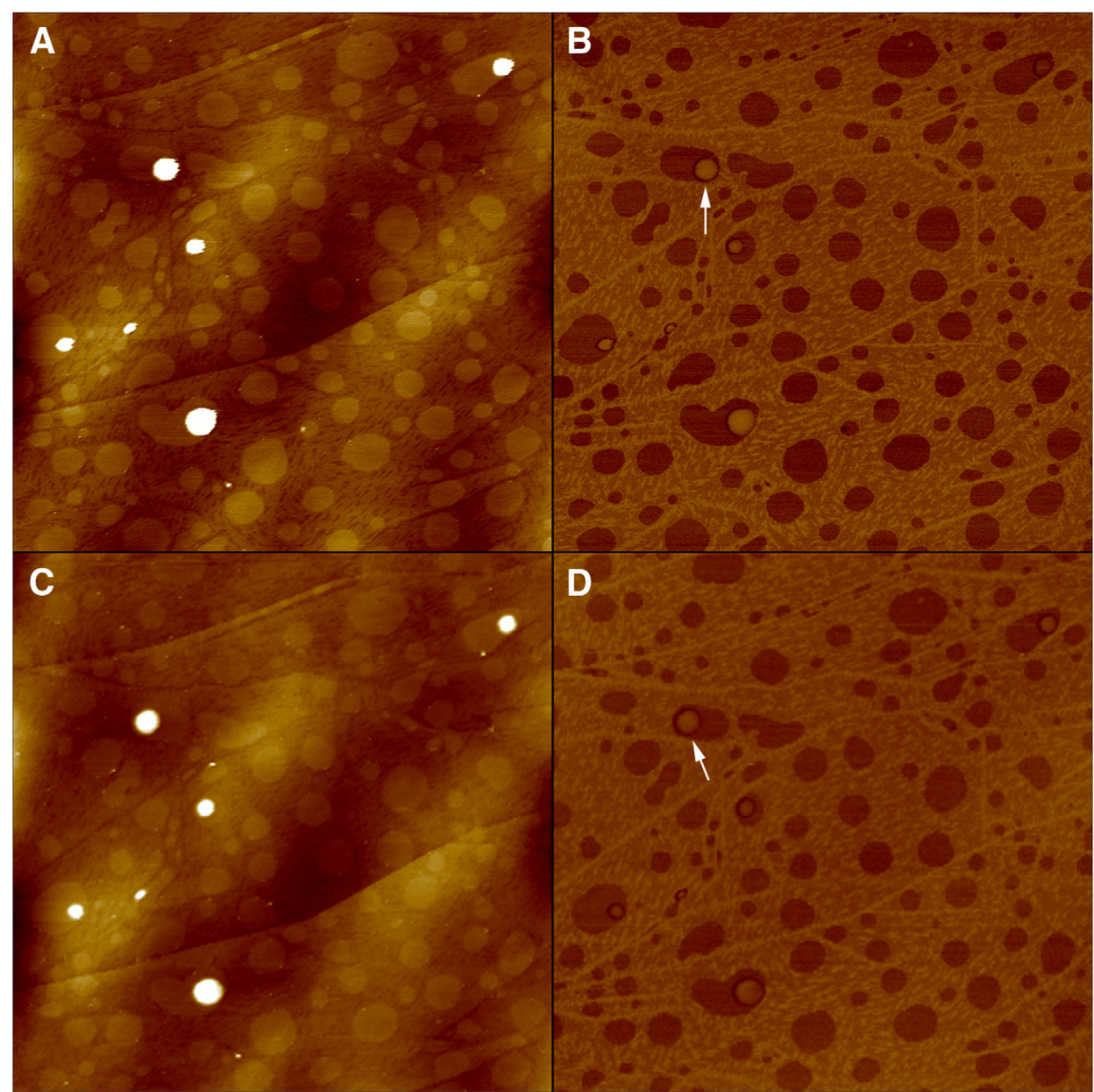

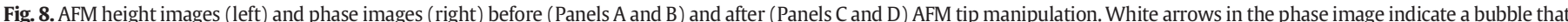

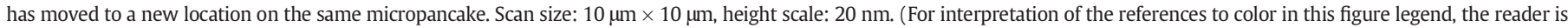
referred to the web version of this article.)

\section{References}

[1] Lou ST, Ouyang ZQ, Zhang Y, Li XJ, Hu J, Li MQ, et al. Nanobubbles on solid surface imaged by atomic force microscopy. J Vac Sci Technol B 2000;18:2573-5.

[2] Ishida N, Inoue T, Miyahara M, Higashitani K. Nanobubbles on a hydrophobic surface in water observed by tapping-mode atomic force microscopy. Langmuir 2000;16: 6377-80.

[3] Parker JL, Claesson PM, Attard P. Bubbles, cavities and the long-ranged attraction between hydrophobic surfaces. J Phys Chem 1994;98:8468-80.

[4] Jin F, Gong XJ, Ye J, Ngai T. Direct measurement of the nanobubble-induced weak depletion attraction between a spherical particle and a flat surface in an aqueous solution. Soft Matter 2008;4:968-71.

[5] Jin F, Li JF, Ye XD, Wu C. Effects of pH and ionic strength on the stability of nanobubbles in aqueous solutions of alpha-cyclodextrin. J Phys Chem B 2007;111: 11745-9.

[6] Jho SH, Kim JY, Song MG, Kim JD. Zeta-potentials of nanobubbles generated by ultrasonication in aqueous solutions, 222. , Abstracts of Papers of the American Chemical Society; 2001 [313-COLL].

[7] Ohgaki K, Khanh NQ Joden Y, Tsuji A, Nakagawa T. Physicochemical approach to nanobubble solutions. Chem Eng Sci 2010;65:1296-300.

[8] Craig VSJ. Very small bubbles at surfaces-the nanobubble puzzle. Soft Matter 2011; 7:40-8.

[9] Epstein PS, Plesset MS. On the stability of gas bubbles in liquid-gas solutions. J Chem Phys 1950;18:1505-9.

[10] Ljunggren S, Eriksson JC. The lifetime of a colloid-sized gas bubble in water and the cause of the hydrophobic attraction. Colloids Surf A 1997;130:151-5.

[11] Ducker WA. Contact angle and stability of interfacial nanobubbles. Langmuir 2009 25:8907-10.

[12] Yang JW, Duan JM, Fornasiero D, Ralston J. Very small bubble formation at the solidwater interface. J Phys Chem B 2003;107:6139-47.

[13] Wildenschild D, Sheppard AP. X-ray imaging and analysis techniques for quantifying pore-scale structure and processes in subsurface porous medium systems. Adv Water Resour 2013;51:217-46.

[14] Marathe R, Turner ML, Fogden A. Pore-scale distribution of crude oil wettability in carbonate rocks. Energy Fuels 2012;26:6268-81.
[15] Pugh RJ. Foaming, foam films, antifoaming and defoaming. Adv Colloid Interf Sci 1996;64:67-142.

[16] Seddon JRT, Lohse D. Nanobubbles and micropancakes: gaseous domains on immersed substrates. J Phys Condens Matter 2011:23.

[17] Zhang LJ, Zhang XH, Fan CH, Zhang Y, Hu J. Nanoscale multiple gaseous layers on a hydrophobic surface. Langmuir 2009;25:8860-4

[18] Zhang XH, Maeda N. Interfacial gaseous states on crystalline surfaces. J Phys Chem C 2011;:115:736-43.

[19] Hampton MA, Nguyen AV. Accumulation of dissolved gases at hydrophobic surfaces in water and sodium chloride solutions: implications for coal flotation. Miner Eng 2009;22:786-92.

[20] Takata Y, Matsubara H, Matsuda T, Kikuchi Y, Takiue T, Law B, et al. Study on line tension of air/hexadecane/aqueous surfactant system. Colloid Polym Sci 2008;286: 647-54.

[21] Law BM. Wetting, adsorption and surface critical phenomena. Prog Surf Sci 2001;66: 159-216.

[22] Zhang X, Maeda N, Craig VSJ. Physical properties of nanobubbles on hydrophobic surfaces in water and aqueous solutions. Langmuir 2006;22:5025-35.

[23] Borkent BM, de Beer S, Mugele F, Lohse D. On the shape of surface nanobubbles. Langmuir 2010;26:260-8.

[24] Song B, Walczyk W, Schonherr H. Contact angles of surface nanobubbles on mixed self-assembled monolayers with systematically varied macroscopic wettability by atomic force microscopy. Langmuir 2011;27:8223-32.

[25] Walczyk W, Schon PM, Schonherr H. The effect of PeakForce tapping mode AFM imaging on the apparent shape of surface nanobubbles. J Phys Condens Matter 2013;25.

[26] Walczyk W, Schonherr H. Closer look at the effect of AFM imaging conditions on the apparent dimensions of surface nanobubbles. Langmuir 2013;29:620-32.

[27] Guillemot L, Biben T, Galarneau A, Vigier G, Charlaix E. Activated drying in hydrophobic nanopores and the line tension of water. Proc Natl Acad Sci U S A 2012; 109:19557-62

[28] Checco A, Guenoun P, Daillant J. Nonlinear dependence of the contact angle of nanodroplets on contact line curvature. Phys Rev Lett 2003;91.

[29] Mendez-Vilas A, Jodar-Reyes AB, Gonzalez-Martin ML. Ultrasmall liquid droplets on solid surfaces: production, imaging, and relevance for current wetting research. Small 2009;5:1366-90. 
[30] Pompe T, Fery A, Herminghaus S. Imaging liquid structures on inhomogeneous surfaces by scanning force microscopy. Langmuir 1998;14:2585-8.

[31] McBride SP, Law BM. Influence of line tension on spherical colloidal particles at liquid-vapor interfaces. Phys Rev Lett 2012:109.

[32] Kameda N, Nakabayashi S. Size-induced sign inversion of line tension in nanobubbles at a solid/liquid interface. Chem Phys Lett 2008;461:122-6.

[33] Zhang XH, Uddin MH, Yang HJ, Toikka G, Ducker W, Maeda N. Effects of surfactants on the formation and the stability of interfacial nanobubbles. Langmuir 2012;28: 10471-7.

[34] van Limbeek MAJ, Seddon JRT. Surface nanobubbles as a function of gas type. Langmuir 2011;27:8694-9.

[35] Weijs JH, Snoeijer JH, Lohse D. Formation of surface nanobubbles and the universality of their contact angles: a molecular dynamics approach. Phys Rev Lett 2012;108.

[36] Peng H, Hampton MA, Nguyen AV. Nanobubbles do not sit alone at the solid-liquid interface. Langmuir 2013;29:6123-30.

[37] Takata Y, Cho JHJ, Law BM, Aratono M. Ellipsometric search for vapor layers at liquid-hydrophobic solid surfaces. Langmuir 2006;22:1715-21.

[38] German SR, Wu X, An H, Craig VSJ, Mega TL, Zhang XH. Interfacial nanobubbles are leaky: permeability of the gas/water interface. ACS Nano 2014;10 [1021/ nn5016049].

[39] Steitz R, Gutberlet T, Hauss T, Klösgen B, Krastev R, Schemmel S, et al. Nanobubbles and their precursor layer at the interface of water against a hydrophobic substrate. Langmuir 2003;19:2409-18.

[40] Lu YH, Yang CW, Hwang IS. Molecular layer of gaslike domains at a hydrophobic-water interface observed by frequency-modulation atomic force microscopy. Langmuir 2012; 28:12691-5.

[41] Gao LC, McCarthy TJ. An attempt to correct the faulty intuition perpetuated by the Wenzel and Cassie "Laws". Langmuir 2009;25:7249-55.

42] de Gennes PG. Wetting - statics and dynamics. Rev Mod Phys 1985:57:827-63.

[43] Butt HJ, Golovko DS, Bonaccurso E. On the derivation of Young's equation for sessile drops: nonequilibrium effects due to evaporation. J Phys Chem B 2007;111: 5277-83.

[44] Zhang LJ, Wang CL, Tai RZ, Hu J, Fang HP. The morphology and stability of nanoscopic gas states at water/solid interfaces. ChemPhysChem 2012;13:2188-95.
[45] Zhang XH, Maeda N, Hu J. Thermodynamic stability of interfacial gaseous states. J Phys Chem B 2008;112:13671-5.

[46] Zhang XH, Zhang XD, Sun JL, Zhang ZX, Li G, Fang HP, et al. Detection of novel gaseous states at the highly oriented pyrolytic graphite-water interface. Langmuir 2007;23: 1778-83.

[47] Atkin R, Craig VSJ, Biggs S. Adsorption kinetics and structural arrangements of cationic surfactants on silica surfaces. Langmuir 2000;16:9374-80.

[48] Atkin R, Craig VSJ, Wanless EJ, Biggs S. Adsorption of 12-s-12 gemini surfactants at the silica-aqueous solution interface. J Phys Chem B 2003;107:2978-85.

[49] Howard SC, Craig VSJ. Very slow surfactant adsorption at the solid-liquid interface is due to long lived surface aggregates. Soft Matter 2009;5:3061-9.

[50] Howard SC, Atkin R, Craig VS]. Effect of electrolyte species on the adsorption of a cationic surfactant to silica: the common intersection point. Colloids Surf A 2009; 347:109-13.

[51] Howard SC, Craig VS]. Adsorption of the cationic surfactant cetyltrimethylammonium bromide to silica in the presence of sodium salicylate: surface excess and kinetics. Langmuir 2009;25:13015-24.

[52] Ducker WA, Senden TJ, Pashley RM. Direct Measurement of Colloidal Forces Using an Atomic Force Microscope. Nature 2006;Vol. 3531991:239-41.

[53] Walsh R, Nelson A, Skinner WM, Parsons D, Craig VSJ. Direct measurement of van der Waals and diffuse double-layer forces between titanium dioxide surfaces produced by atomic layer deposition. J Phys Chem C 2012;116:7838-47.

[54] Velegol SB, Fleming BD, Biggs S, Wanless EJ, Tilton RD. Counterion effects on hexadecyltrimethylammonium surfactant adsorption and self-assembly on silica. Langmuir 2000;16:2548-56.

[55] Colas A. Siang J, Ulman K. Silicones in pharmaceutical applications. Part 5: Siliconization of Parenteral Packaging Components. Midland: Dow Corning Corporation; 2006 1-5.

[56] Berkelaar RP, Dietrich E, Kip GAM, Kooji ES, Zandvliet HJW, Lohse D. Exposing nanobubble-like objects to a degassed environment. Soft Matter 2014:10:4947-55.

[57] Zhang XH, Ducker W. Formation of interfacial nanodroplets through changes in solvent quality. Langmuir 2007;23:12478-80.

[58] Zhang XH, Wei XX, Ducker W. Formation of nanodents by deposition of nanodroplets at the polymer-liquid interface. Langmuir 2010;Vol. 26(7):4776-81. 